The Bangladesh Veterinarian (2008) 25(1) : 9 - 16

\title{
Caprine lung diseases and causal bacteria
}

\author{
T. Ferdausi, M. G. Haider, K. J. Alam³, M. A. Baki and M. M. Hossain*
}

Department of Pathology, Faculty of Veterinary Science, Bangladesh Agricultural University, Mymensingh-2202, Bangladesh

\begin{abstract}
Pathological conditions in lungs of slaughtered goats were studied. Sixty lungs were examined and tissue samples and swabs obtained for histopathology and bacterial isolation, respectively. The prevalence of lung diseases was $58.3 \%(n=35)$. Gross lesions were categorized into: (a) haemorrhage and congestion 25\% (b) emphysema $21.7 \%$ (c) hepatization $3.3 \%$ and (d) granulomatous nodules about $1 \mathrm{~mm}$ diameter $8.3 \%$. On histopathological examination, 10 types of lesions were found: (a) bronchitis $6.7 \%$, (b) small cell anaplastic carcinoma 3.3\%, (c) pneumonia 6.7\%, (d) interstitial pneumonia 15\%, (e) emphysema $6.7 \%$, (f) bronchopneumonia 3.3\%, (g) purulent pneumonia 5\%, (h) haemorrhagic pneumonia $3.3 \%$, (i) pulmonary adenomatosis $1.7 \%$ and (j) no lesions $6.7 \%$. Pasteurella sp. (11.7\%), Escherichia coli (E. coli; 6.7\%), Staphylococcus sp. (36.7\%) and Bacillus sp. (3.3\%) were isolated from the lungs. Pasteurella sp. was found in haemorrhagic pneumonia, interstitial pneumonia, small cell anaplastic carcinoma and bronchitis, followed by Bacillus sp. in haemorrhagic pneumonia, E. coli in interstitial pneumonia and pulmonary adenomatosis and Staphylococcus sp. from emphysema, bronchopneumonia, pneumonia, bronchitis and purulent pneumonia. (Bangl. vet. 2007. Vol. 25, No. 1, 9-16)
\end{abstract}

\section{Introduction}

The goat is an important species of livestock in third world countries. Bangladesh, a tropical agro-based developing country, possesses the third largest population in Asia of over 34 million goats (FAO, 2007). This represents over $57 \%$ of the total livestock population. Over $90 \%$ of the goats belong to the Black Bengal breed.

Lungs are vulnerable to many infectious agents. Pneumonia causes debility and death leading to great economic loss to farmers. Women commonly rear a few goats, grazed usually on free pastures. These pastures are usually contaminated with infectious agents that can get access through inhalation and cause pneumonia. A large number of goats are brought to the Veterinary Clinic or Hospital for treatment of respiratory problems, especially pneumonia. A variety of causes are responsible for pneumonia in goats (Rahman et al., 1976). This investigation was undertaken to determine the status of lung diseases in Black Bengal goats.

\footnotetext{
3 Department of Pathology and Parasitology, Barisal Government Veterinary College, Babuganj, Barisal, Bangladesh

* Correspondence :- E-mail : mmhossain 04 @ yahoo.com.au
} 


\section{Materials and Methods}

\section{Samples}

Lung samples were collected immediately after slaughter from four abattoirs in Mymensingh sadar upazila (sub-district) from May to September 2007. Out of 60 lungs examined 35 were affected, and were collected for pathological studies: 35 swabs were collected aseptically from inner core of lungs, and immediately placed in nutrient broth for bacteriology. All samples were transferred to the laboratory for histopathology and bacteriology.

\section{Pathological studies}

Gross : Sixty lungs were examined for gross abnormalities, which were recorded, and representative tissue samples containing lesions were fixed in $10 \%$ neutral buffered formalin for histopathology.

Histopathology: The fixed tissues were processed as described by Luna (1968).

\section{Photomicrography}

Photomicrography was done using an Olympus PM-C 35 camera.

\section{Isolation and identification of bacteria}

Test tubes containing samples were incubated for 24 hours at $37^{\circ} \mathrm{C}$. From the nutrient broth, subcultures were done on blood agar, MacConkey agar, EMB agar, TSI agar and nutrient agar, and incubated at $37^{\circ} \mathrm{C}$ overnight. The identification of the organisms was done as described by Freeman (1985) and Cheesbrough (2000). The organisms were isolated and identified on the basis of colony and staining characteristics and biochemical tests.

\section{Biochemical tests}

Catalase activity : Organisms were grown on a slope of nutrient agar. One $\mathrm{ml} 3 \%$ hydrogen peroxide was run down the slope and examined after $5 \mathrm{~min}$ for evolution of gases.

Coagulase test : Undiluted plasma $(0.5 \mathrm{ml})$ was mixed with an equal volume of old broth culture for $18-24$ hour at $37^{\circ} \mathrm{C}$ for 4 hours and examined after 1 and 4 hours for a coagulum. Negative tubes were left at room temperature overnight and then reexamined.

Indole test : Peptone water $(2 \mathrm{ml})$ was inoculated with $5 \mathrm{ml}$ of bacterial culture and incubated for 48 hours. Kovac's reagent $(0.5 \mathrm{ml})$ was added, shaken well and examined after one minute. Formation of a red layer was the indication of indole positive.

Methyl- red test: A colony of the test organism was inoculated in $0.5 \mathrm{ml}$ of sterile glucose phosphate broth (as used in the V-P test). After overnight incubation at $35-37^{\circ} \mathrm{C}$, a drop of methyl red solution was added. A positive reaction was shown by 
a bright red colour, indicating acidity. A yellow or orange colour was indicated as negative.

\section{Maintenance of stock culture}

To preserve organisms, they were inoculated at $37^{\circ} \mathrm{C}$ for 24 hours. After growth of the organisms the tubes were sealed with paraffin wax and kept at $4^{\circ} \mathrm{C}$.

\section{Results and Discussion}

The prevalence of gross lung lesions was $58.3 \%$, categorized into haemorrhages and congestion 25\%, emphysema 21.7\%, hepatization $3.3 \%$ (Fig. 1) and granulomatous nodules lesions about $1 \mathrm{~mm}$ diameter $8.3 \%$.

Histologically, lesions were categorized into 10 types: bronchitis $6.7 \%$, small cell anaplastic carcinoma 3.3\%, pneumonia $6.7 \%$, interstitial pneumonia $15 \%$, emphysema $6.7 \%$, bronchopneumonia $3.3 \%$, purulent pneumonia $5 \%$, haemorrhagic pneumonia $3.3 \%$, pulmonary adenomatosis $1.7 \%$ and no apparent lesions $6.7 \%$ (Table 2).

Bronchitis : No specific gross lesions were found but the consistency of lung was hard on palpation. Microscopically, there was exudate in most of the lumen of the bronchus. Infiltration of neutrophils was found in the lumen and wall of the bronchus (Fig. 3).

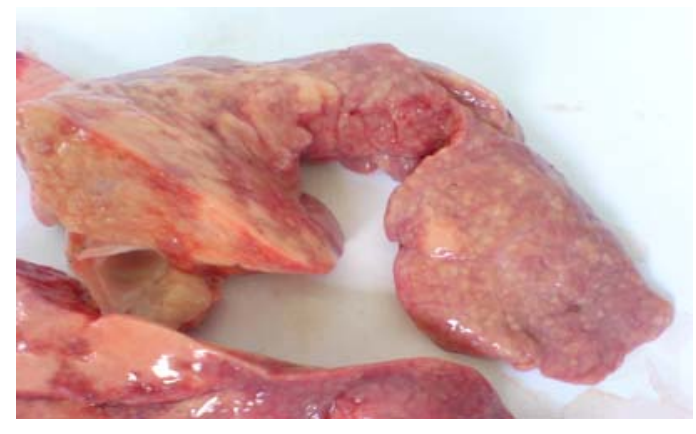

Fig. 1. Caprine lungs showing grey hepatization

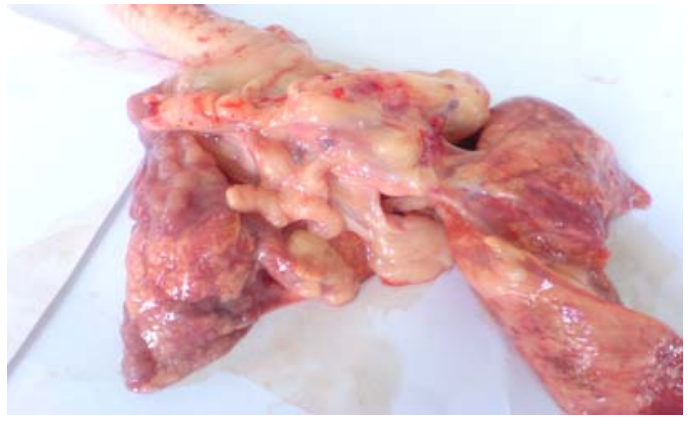

Fig. 2. Caprine lungs showing small nodule

Small cell anaplastic carcinoma : Grossly, the affected portion of lung showed consolidation. Microscopically, the epithelium was proliferated from the bronchial wall causing hypercellularity and loss of normal structure. The neoplastic cells consisted of single layer of cuboidal or low columnar epithelial cells with uniform shape and size.

Pneumonia: Grossly, there was haemorrhage and in some cases congestion. Excessive haemorrhages within the alveoli and inter-alveolar septa associated with leukocytic infiltration was found histologically. Similar infiltrations were noticed in 
the bronchus. The wall of the bronchus showed inflammatory changes and mild necrosis in the mucosal layer.

Table 1. Gross pathology of goat lungs

\begin{tabular}{llcc}
\hline \multicolumn{2}{c|}{ Lungs lesions } & No. of affected animal & Disorder (\%) \\
\hline a. Haemorrhage and congestion in lung & 15 & 25 \\
b. & Emphysematous lung & 13 & 21.7 \\
c. Hepatization in lung & 2 & 3.3 \\
d. $\quad$ Granulomatous nodule like lesions in & 5 & 8.3 \\
$\quad$ lung & & \\
\hline \multicolumn{2}{l}{ Total } & 35 & 58.3 \\
\hline
\end{tabular}

Table 2. Histopathology of goat's lung $(n=60)$

\begin{tabular}{ll|c|c}
\hline & \multicolumn{1}{c|}{ Lungs lesions } & No. of animal affected & Disorder $(\%)$ \\
\hline a. & Bronchitis & 4 & 6.7 \\
b. & Small cell anaplastic carcinoma & 2 & 3.3 \\
c. & Pneumonia & 4 & 6.7 \\
d. & Interstitial pneumonia & 9 & 15 \\
e. & Emphysema & 4 & 6.7 \\
f. & Bronchopneumonia & 2 & 3.3 \\
g. & Purulent pneumonia. & 3 & 5.0 \\
h. & Haemorrhagic pneumonia & 2 & 3.3 \\
i. & Pulmonary adenomatosis & 1 & 1.7 \\
j. & No lesions & 4 & 6.7 \\
\hline
\end{tabular}

Interstitial pneumonia : Grossly, the lungs were reddish with small dark-red spots, which were hard on palpation. Microscopically, the alveolar septa were thickened due to accumulation of macrophages and lymphocytes with proliferation of fibrous connective tissue (Fig. 4).

Emphysema of lungs : The emphysematous area was pale in colour with elevated areas that were easily detected by compression with a finger. Many alveoli were distended with wide openings due to rupture of alveolar walls. The alveolar wall was thin and atrophic under the microscope.

Broncho-pneumonia : Grossly, the affected portion of lung was congested, consolidated and hard on palpation. Microscopically, there was exudation with infiltration of neutrophils in the bronchiolar wall and in the lumen of bronchioles. There was deposition of exudates within the peribronchial alveoli. 
Purulent pneumonia: Grossly, the lung was consolidated and hard. Microscopic lesions were characterized by inflammatory cells, mainly neutrophils, within the alveoli and in the lumen of the bronchioles. Adjacent to the bronchioles, there were focal infiltrations of a large number of neutrophils and a few lymphocytes.

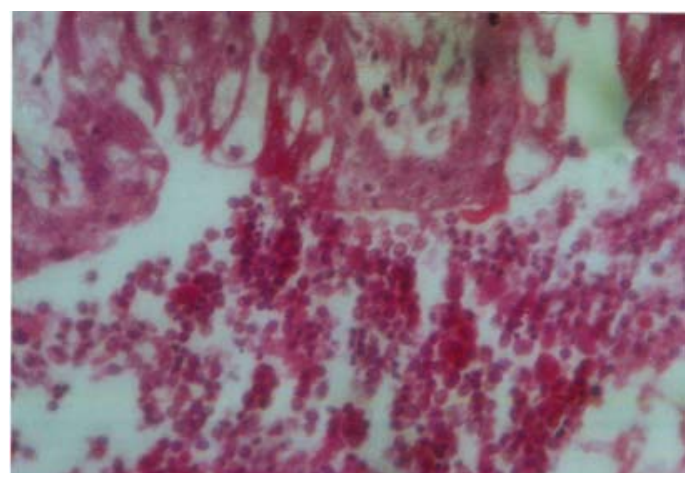

Fig. 3. Section of caprine lung showing bronchitis: Note exudate in most of lumen of the bronchus and infiltration of inflammatory cells mainly neutrophils in and around the bronchial wall (H\&E, $x 333$ )

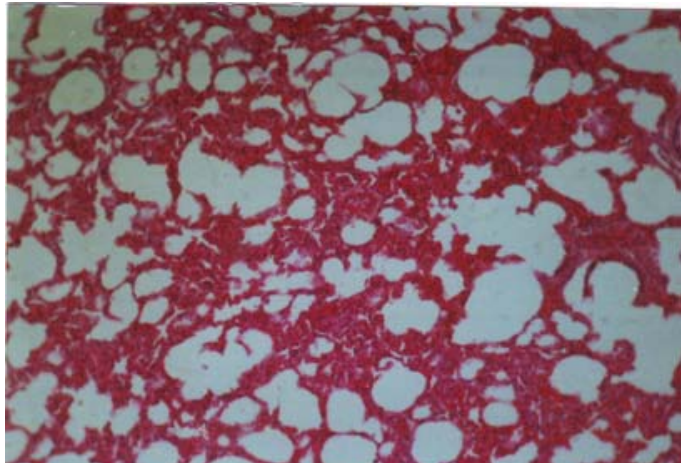

Fig. 4. Section of caprine lung showing interstitial pneumonia: Note the alveolar septa is thickened due to accumulation of macrophages and lymphocytes (H\&E, $x$ 830)

Haemorrhagic pneumonia: The lungs showed severe congestion. There were many microscopic haemorrhages within the alveoli and inter-alveolar septa, associated with leukocytic infiltration. Haemorrhages and leukocytic infiltration were found in the bronchus. The wall of the bronchus showed inflammatory change.

Pulmonary adenomatosis: No specific gross lesions were found but the consistency of lung was firmer on palpation. Glandular pattern of growth due to pneumocyte Type-II cells proliferation was found histologically.

Pus and cyst in lungs were recorded by Jubb et al. (1993); Radostits et al. (2002), but no such lesions were recorded in the present investigation.

The highest occurrence of microscopic lung lesions was interstitial pneumonia (15\%) where Pasteurella sp. and E. coli were present. The role of these bacteria in the production of the lesion was not clear. However, the interstitial pneumonic lesions were characterized by thickening of inter-alveolar septa with macrophages, lymphocytes with proliferation of fibrous connective tissue. The histopathological lesions of interstitial pneumonia correspond with the findings of Jones et al. (1997); Zamri-Saad (2006). Histopathology of broncho-pneumonia, haemorrhagic pneumonia and pulmonary adenomatosis described in the present investigation were similar to those of Akbar (2007); Jones et al. (1997). 


\section{Bacterial isolation and identification}

Among the 60 lungs, seven were positive for Pasteurella sp., four for E. coli, 22 for Staphylococcus sp. and two for Bacillus sp. (Table 3). Pasteurella sp. was isolated from haemorrhagic pneumonia, interstitial pneumonia, small cell anaplastic carcinoma and bronchitis. Bacillus sp. was isolated from haemorrhagic pneumonia. E. coli from interstitial pneumonia and pulmonary adenomatosis was isolated. Staphylococcus sp. from emphysema, bronchopneumonia, pneumonia, bronchitis and purulent pneumonia was found. The prevalence of Pasteurella sp. was $11.7 \%$, E. coli $6.7 \%$, Staphylococcus sp. 36.7\% and Bacillus sp. 3.3\% (Table 3).

Table 3. Detection of bacteria in the lung $(n=60)$

\begin{tabular}{l|c|c|c}
\hline \multicolumn{1}{c|}{ Finding } & $\begin{array}{c}\text { Total No. sample } \\
\text { examined }\end{array}$ & $\begin{array}{c}\text { No. of affected } \\
\text { animal }\end{array}$ & $\begin{array}{c}\text { Bacteria isolated } \\
(\%)\end{array}$ \\
\hline Pasteurella sp. & 60 & 7 & 11.7 \\
E. coli & & 4 & 6.7 \\
Staphylococcus sp. & & 22 & 36.7 \\
Bacillus sp & & 2 & 3.3 \\
\hline Total & 60 & 35 & 59.4 \\
\hline
\end{tabular}

Isolation and identification of Pasteurella sp.

Pasteurella sp. grew well on nutrient agar and showed smooth, circular, greyish colony about $1 \mathrm{~mm}$ in diameter after 24 hour at $37^{\circ} \mathrm{C}$. In nutrient broth, they grew well and showed diffuse turbidity. Small greyish haemolytic zones were evident in blood agar. In McConkey agar, they showed small pink colonies about 1-2 $\mathrm{mm}$ in diameter. Smear from pure culture revealed Gram-negative, bipolar, small rodshaped bacteria. In Leishman's stain bipolar characteristics of the organisms were detected with a blue colour (Fig. 5). Pasteurella sp. was catalase-positive and indolenegative. Pasteurella sp. fermented sucrose, dextrose and maltose with the production of acid but did not ferment lactose.

\section{Isolation and identification of Staphylococcus sp.}

Staphylococcus sp. grew well on nutrient agar and showed smooth circular, opaque often yellow-pigmented colonies about $1 \mathrm{~mm}$ in diameter after 24 hour at $37^{\circ} \mathrm{C}$. In nutrient broth, they grew well and showed dense turbidity with a powdery deposit. Smears from pure cultures revealed Gram-positive, round bacteria in cluster form.

\section{Isolation and identification of Escherichia coli}

The organism produced smooth circular colonies with dark centres and metallic sheen on EMB agar. E. coli produced pink colonies on MacConkey's agar. E. coli was a short rod, varying from coccoid bipolar shapes to long filamentous forms. It was occurred singly or in short Gram-negative chains. E. coil fermented dextrose, lactose, 
maltose, mannitol, sucrose and dulcitol. The organisms were MR positive, VP negative and produced indole.

\section{Isolation and identification of Bacillus sp.}

Bacillus sp. grew readily on blood agar. On agar they produced haemolytic zone. The bacilli were Gram-negative, rod-shaped cells in chain form (Fig. 6).

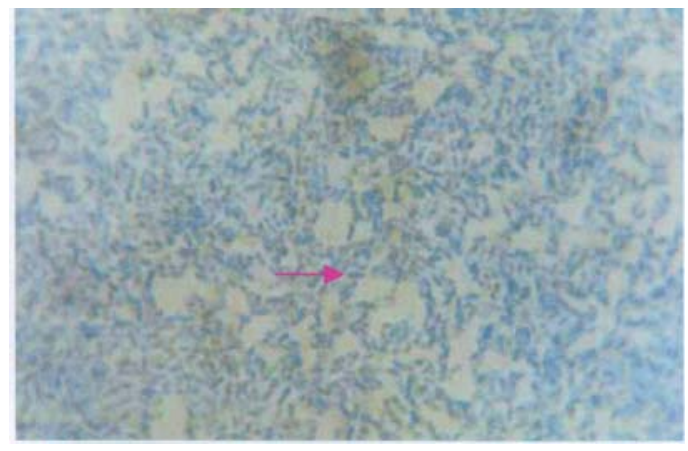

Fig. 5. Smear from pure culture of Pasteurella $s p$. stained with Leishman's stain : Note the Gram-negative, rod-shaped blue bacteria $(x 830)$

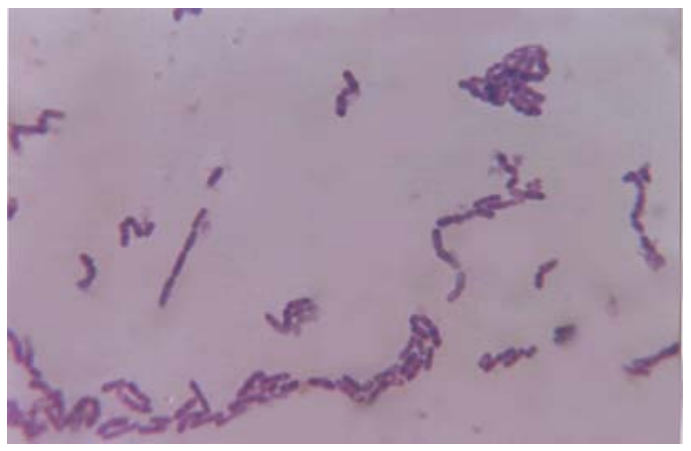

Fig. 6. Smear from pure culture of Bacillus sp. is stained with Gram's stain: Note Gramnegative, rods in chain form (x 830)

Pneumonia (chronic interstitial pneumonia, broncho-pneumonia, congested stage of pneumonia, fibrinous pneumonia, purulent pneumonia) are caused by Pasteurella multocida (Shafarin et al., 2007; Zamri-saad et al., 2006); Pasteurella haemolytica (Brogden et al., 1998); Corynebacterium sp. (Sharma and Dwivedi, 1977); Staphylococcus sp. and Streptococcus sp. (Almeida et al., 1986; Ugochukwu, 1986) and Escherichia coli (Kapur et al., 1976). However, Pasteurella sp. (11.7\%), Escherichia coli (6.7\%), Staphylococcus sp. (36.7\%) and Bacillus sp. (3.3\%) were identified.

It cannot be clearly explained the role of bacterial agents in the development of lung lesions. Neoplasms (Archer et al., 2007; De Las Heras et al., 2006) have also been reported. However, no attempt was made to characterize other aetiological agents except bacteria that warrant further study.

\section{References}

Akbar M 2000: Pathological investigation on trachea and lung lesions of buffaloes in Bangladesh. MS Thesis, Department of Pathology, Bangladesh Agricultural University, Mymensingh.

Archer F, Jacquier E, Lyon M, Chastang J, Cottin V, Mornex JF, Leroux C 2007: Alveolar type II cells isolated from pulmonary adenocarcinoma: a model for JSRV expression in vitro. American Journal of Respiratory and Cell Molecular Biology 36 534-40.

Brogden KA, Lehmkuhl HD, Cutlip RC 1998: Pasteurella haemolytica complicated respiratory infections in sheep and goats. Veterinary Research 29 233-54. 
BSS 2007: Massive initiative for goat rearing undertaken in northern Bangladesh, Bangladesh Sangbad Sangshtha, Dhaka, Bangladesh.

Cheesbrough M 2000: District Laboratory Practice in Tropical Countries. Part-2. Cambridge University Press, UK, pp. 64-65.

De las Heras M, Ortin A, Benito A, Summers C, Ferrer LM, Sharp JM 2006: In-situ demonstration of mitogen-activated protein kinase Erk1/2 signalling pathway in contagious respiratory tumours of sheep and goats. Journal of Comparative Pathology 135 1-10.

De las Heras, M., González L, Sharp JM 2003: Pathology of ovine pulmonary adenocarcinoma. Current Topical Microbiology and Immunology 275 25-54.

FAO 2007: Black Bengal promising goat genetic resource of Bangladesh, Food and Agriculture Organization of the United Nation, Rome, Italy.

Freeman JN 1985: Burrow's Text Book of Microbiology. 22nd edn. WB Saunders Company, London, UK, pp. 372-472.

Jones TC, Hunt RD, King NW 1997: Veterinary Pathology. $6^{\text {th }}$ edn, Williams and Wilkins, England pp. 345.

Jubb KVF, Kennedy PC, Palmer N 1993: Pathology of Domestic Animals. $4^{\text {th }}$ edn, Vol. 2, Academic Press, Inc pp. 638.

Kapur MP, Sadana JR, Kalra DS, Chauhan HVS 1976: Some observations on the causes of lamb and kid mortality at the Haryana Agricultural University, Hissar. Veterinary Bulletin 461775.

Luna LG 1968: Manual of Histologic Staining Methods of the Armed Forces Institute of Pathology. $3^{\text {rd }}$ end. Mcgraw Hill Book Co. New York.

Rahman A, Ahmed MU, Mia AS 1976: Studies on the diseases of goats in Bangladesh. Mortality of goats under farm and rural conditions. Tropical Animal Health and Production 890.

Shafarin MS, Zamri-Saad M, Jamil SM, SitiKhairani B, Saharee AA 2007: Experimental transmission of Pasteurella multocida 6: B in goats. Journal of Veterinary Medicine: A Physiological Pathological Clinical Medicine 54 136-9

Sharma DN, Dwivedi JN 1977: Pseudotuberculosis lesions in lung of sheep and goats. Veterinary Bulletin 476054.

Zamri SM, Effendy AWM, Maswati MA, Salim N, Sheikh OAR 1996: The goat as a model for studies of pneumonia pasteurellosis caused by Pasteurella multocida. British Veterinary Journal 152 453-458.

Zamri-Saad M, Ernie ZA, Sabri MY 2006: Protective effect following intranasal exposure of goats to live Pasteurella multocida B:2. Tropical Animal Health and Production $\mathbf{3 8}$ 541-6. 